\title{
THE ATKINSON-WILCOX EXPANSION THEOREM FOR ELASTIC WAVES*
}

\author{
BY \\ GEORGE DASSIOS ${ }^{1}$ \\ University of Patras, Greece
}

\begin{abstract}
Consider the problem of scattering of an elastic wave by a three-dimensional bounded and smooth body. In the region exterior to a sphere that includes the scatterer, any solution of Navier's equation that satisfies the Kupradze's radiation condition has a uniformly and absolutely convergent expansion in inverse powers of the radial distance from the center of the sphere. Moreover, the coefficients of the expansion can recurrently be evaluated from the knowledge of the leading coefficient, known as radiation pattern. Therefore, a one-to-one correspondence between the scattered fields and the corresponding radiation patterns is established. The acoustic and electromagnetic cases are recovered as special cases.
\end{abstract}

1. Introduction. The Sommerfeld's radiation condition [17] imposes, upon any scalar scattered field, the appropriate asymptotic characteristics in order for the scattering problem to have a unique solution. It actually states that the behaviour of the scattered field, far away from the scatterer, should coincide with the behaviour of an outgoing spherical wave emanating from an oscillatory point source. Müller [13] and Silver [16] provided the corresponding radiation condition for electromagnetic scattering, while in the case of elastic wave scattering, the appropriate radiation conditions for the longitudinal as well as the transverse waves are due to Kupradze [8]. Stoker [18], [19] attempted to replace the radiation condition, which is actually imposed upon the spatial behaviour of the time-independent wave solution, with appropriate initial conditions imposed upon the (time-dependent) solution of the wave equation, and then find the solution of the time-independent problem by passing to the limit as time tends to infinity. He illustrated his idea by a few examples taken from hydrodynamics. Wilcox [26] put this idea in general framework, by showing that Sommerfeld's radiation condition for a time-harmonic wave is a consequence of the fact that a solution of the initial-boundary value problem represents a wave propagating outward from the source (scatterer) with a constant velocity. His method is based on the concept of spherical means.

It was Atkinson [1], in 1949, who first realized that the Sommerfeld's asymptotic condition has an exact counterpart in terms of a convergent series representation

*Received December 31, 1986.

' Visiting the Department of Mathematics, University of Tennessee, Knoxville, TN 37996-1300.

(C) 1988 Brown University 
of the scattered field in the exterior of any sphere completely surrounding the scatterer. In fact, Atkinson showed that any solution of the Helmholtz's equation, which satisfies the radiation condition, has an absolutely and uniformly convergent series representation in inverse powers of the radial distance $r$, in all space exterior to the above sphere. The coefficients of this expansion depend only on direction. The leading term of the series has the form of the Rayleigh's radiation condition [15], [23], which is nothing else but the fundamental solution of the time-independent wave equation whose form reflects the rotational invariance of Laplace's operator [6]. Atkinson's expansion separates the radial from the angular dependence of the solution and it can be seen as the wave analogue of Maxwell's multiple expansion [12] for potential functions due to spatially localized sources. In this radial-angular separation, the radial dependence is the same in all scattering problems and characterizes the radiative nature of the scattered field, while the angular dependence incorporates the geometrical and the physical characteristics of each particular scatterer. Atkinson's expansion implies that the periodic variations carried by any term of the series have all the same form, but their amplitudes become smaller and smaller as we move to higher and higher order terms in the series.

A few years later, Wilcox published two fundamental papers generalizing the idea of radiation condition from a pointwise limit to an $L^{2}$-type limit, hence weakening the sufficient conditions for an integral representation of the scattered field and the Atkinson's expansion theorem, both for the acoustic [24], as well as the electromagnetic [25] case. A crucial step in generalizing the Müller-Silver radiation condition for electromagnetic waves, to the corresponding $L^{2}$-form was an integral identity for electromagnetic waves proved by Stratton and Chu [20]. Wilcox's work contains the important fact that all the (orientation dependent) coefficients in the Atkinson's expansion can be recovered through a recurrence relation, via the leading coefficient, known as scattering amplitude [22], or radiation pattern [14]. Therefore, a oneto-one correspondence between the scattered fields and their radiation patterns was established. A closed form expression for the scattered field in terms of the normalized scattering amplitude, in the scalar case, has been obtained by Twersky [22]. Twersky's expansion formula is based upon the development of the spherical Hankel functions in inverse powers of the radial variable. The expansion theorem brought up the question of characterizing all the functions defined on the unit sphere that can be radiation patterns for some scattered fields. The answer to this question was given by Müller [14] who proved that a necessary and sufficient condition in order for a given function to be a radiation pattern is that it is the restriction on the unit sphere of an entire harmonic function, whose $L^{2}$-norm over a sphere of radius $R$ is a function of exponential type in the variable $R$.

The two-dimensional version of Atkinson's theorem for acoustic waves was proved by Karp [7] in a form that involves a combination of two series expansions in inverse powers of the radial distance. One expansion is proportional to the Hankel function $H_{0}$ and the other is proportional to the Hankel function $H_{1}$.

The present work studies the elastic scattering problem from the point of view of representing the scattered field by an Atkinson type expansion, outside a sphere 
that includes the scatterer. Section 2 contains a short description of the scattering problem in classical elasticity. The main expansion theorem is stated and proved in Section 3. In order to facilitate reading, the proof of the theorem has been reduced to a small number of lemmas. The scattered elastic field is a linear combination of two series in inverse powers of the radial variable $r$. One involves the longitudinal part and the other involves the transverse part of the scattered wave. The radial dependence of both series is scalar, while the vectorial character of the elastic displacement field is reflected in the coefficients of the expansion which are dependent only on the orientation of the observation point. The convergence properties of both series in the expansions are proved to be the same as those of the scalar case. Section 4 involves the consequences of the expansion theorem. It is actually proved that all the coefficients of the series that correspond to the longitudinal part can be evaluated from the knowledge of the coefficient of the leading term of the longitudinal series alone. The same is true for the transverse wave. Therefore, a one-to-one correspondence between the longitudinal parts of the displacement fields and the longitudinal radiation patterns, on one hand, and between the transverse parts of the displacement fields and the transverse radiation patterns on the other, is established. In contrast to the acoustic and electromagnetic scattering problems, where the recurrence relations express the $n$-th coefficient of the series in terms of the $(n-1)$-th coefficient, in the problem of elastic scattering, both for the longitudinal as well as for the transverse series, the first-order coefficients are given in terms of the corresponding zeroth order, while all the $n$-th order coefficients for $n \geq 2$ are given in terms of the $(n-1)$-th and $(n-2)$-th order coefficients. This is a consequence of the more complicated structure of Navier's equation as compare with the scalar and vector wave equation. We also obtain the well-known result, [2], [4], that the longitudinal radiation pattern has a radial direction while the transverse radiation pattern is tangentially polarized. These polarization properties yield some calculational difficulties in evaluating the recurrence relations for the coefficients, as can be seen in the proof of Proposition 1. Furthermore, we show how the coefficients of the longitudinal and the transverse expansion can be expressed via the normalized longitudinal and transverse scattering amplitudes, which are given in [4], for each elastic scattering problem, in terms of quadratures over the surface of the scatterer. Finally, in Sec. 5, we recover, as special cases, the Atkinson-Wilcox theorems for acoustic as well as for electromagnetic waves and show how the third-order recurrence relation of the elastic case reduces to the corresponding second-order recurrence relations obtained by Wilcox.

2. The elastic scattering problem. . Let the scatterer $V^{-}$be a bounded, convex, and closed subset of $\mathbf{R}^{3}$, with a smooth boundary $S$. The complement $V$ of the region $V^{-}$is occupied by a homogeneous and isotropic elastic medium characterized by the constant mass density $\rho$ and the Lamé constants $\lambda$ and $\mu$. We assume that infinite time has passed since the application of the initial disturbance, so that the transient wave [26] has died out and the purely time harmonic dependence $e^{-i \omega t}$ has been established. Then the time-dependent displacement field $\mathbf{u}(\mathbf{r})$ satisfies the stationary Navier equation

$$
\mu \Delta \mathbf{u}+(\lambda+\mu) \nabla(\nabla \cdot \mathbf{u})+\rho \omega^{2} \mathbf{u}=\mathbf{0},
$$


where $\omega$ is the angular frequency imposed by the assumed time harmonic dependence. In what follows, the mass density $\rho$ has been scaled out to unity.

The total field $\boldsymbol{\Psi}=\boldsymbol{\Phi}+\mathbf{u}$, where $\boldsymbol{\Phi}$ denotes the incident field and $\mathbf{u}$ the scattered field, has to satisfy specific boundary conditions on $S$. Among these, the most important is the boundary condition for a rigid scatterer: $\mathbf{u}=\mathbf{0}$, on $S$, and the boundary condition for a cavity: $T \mathbf{u}=\mathbf{0}$ on $S$. The surface traction operator $T$ is defined to be [9]

$$
T=2 \mu \hat{\mathbf{n}} \cdot \nabla+\lambda \hat{\mathbf{n}} \operatorname{div}+\mu \hat{\mathbf{n}} \times \operatorname{rot},
$$

where $\hat{\mathbf{n}}\left({ }^{2}\right)$ is the outward unit normal on $S$, which exists everywhere by the smoothness of $S$. The field $\mathbf{u}$ is linearly decomposed [21] into the longitudinal wave $\mathbf{u}^{p}$, which propagates with the phase velocity $c_{p}=\sqrt{\lambda+2 \mu}$, and the transverse wave $\mathbf{u}^{s}$, which propagates with the phase velocity $c_{s}=\sqrt{\mu}$, i.e.,

$$
\mathbf{u}(\mathbf{r})=\mathbf{u}^{p}(\mathbf{r})+\mathbf{u}^{s}(\mathbf{r}) \text {. }
$$

The longitudinal wave $\mathbf{u}^{p}$ and the transverse wave $\mathbf{u}^{s}$ satisfy the Kupradze radiation conditions [8]

$$
\begin{gathered}
\lim _{r \rightarrow \infty} \mathbf{u}^{p}(\mathbf{r})=\lim _{r \rightarrow \infty} r\left(\partial_{r} \mathbf{u}^{p}(\mathbf{r})-i k_{p} \mathbf{u}^{p}(\mathbf{r})\right)=\mathbf{0}, \\
\lim _{r \rightarrow \infty} \mathbf{u}^{s}(\mathbf{r})=\lim _{r \rightarrow \infty} r\left(\partial_{r} \mathbf{u}^{s}(\mathbf{r})-i k_{s} \mathbf{u}^{s}(\mathbf{r})\right)=\mathbf{0},
\end{gathered}
$$

uniformly over directions, where $k_{p}$ and $k_{s}$ are the wave numbers of the longitudinal and the transverse waves respectively, which are related via

$$
\omega=c_{p} k_{p}=c_{s} k_{s} \text {. }
$$

The direct scattering problem in elasticity is the following. Given an incident field $\boldsymbol{\Phi}$ and boundary conditions on $S$, find the scattered field $\mathbf{u}$ which satisfies Eq. (1), the appropriate boundary conditions and the radiation conditions (4), (5). The solution of this problem is furnished via the integral representation, [9], [4],

$$
\mathbf{u}(\mathbf{r})=\frac{1}{4 \pi} \int_{S}\left[\Psi\left(\mathbf{r}^{\prime}\right) \cdot T_{r^{\prime}} \boldsymbol{\Gamma}\left(\mathbf{r}, \mathbf{r}^{\prime}\right)-\Gamma\left(\mathbf{r}, \mathbf{r}^{\prime}\right) \cdot T_{r^{\prime}} \Psi\left(\mathbf{r}^{\prime}\right)\right] d S\left(\mathbf{r}^{\prime}\right),
$$

which holds for every $\mathbf{r} \in V$. The fundamental tensor $\Gamma\left(\mathbf{r}, \mathbf{r}^{\prime}\right)$ is given by

$$
\boldsymbol{\Gamma}\left(\mathbf{r}, \mathbf{r}^{\prime}\right)=-\frac{1}{\omega^{2}} \nabla_{\mathbf{r}} \otimes \nabla_{\mathbf{r}} \frac{e^{i k_{p}\left|\mathbf{r}-\mathbf{r}^{\prime}\right|}}{\left|\mathbf{r}-\mathbf{r}^{\prime}\right|}+\frac{1}{\omega^{2}}\left[\nabla_{\mathbf{r}} \otimes \nabla_{\mathbf{r}}+k_{s}^{2} \mathbf{I}\right] \frac{e^{i k_{s}\left|\mathbf{r}-\mathbf{r}^{\prime}\right|}}{\left|\mathbf{r}-\mathbf{r}^{\prime}\right|}
$$

where $\mathbf{I}$ is the identity dyadic. Besides its theoretical importance, this representation has also a significant computational value since it reduces a three-dimensional problem over the unbounded region $V$ to a two-dimensional problem over the bounded surface $S[3]$.

3. The expansion theorem. Following the elegant work of Wilcox, [24], [25], we give the following definitions in order to simplify the statements of the theorems.

Definition 1. Let $V$ be the exterior domain described in Sec. 2. The vector field $\mathbf{u}: V \rightarrow \mathbf{C}^{3}$ is called an exterior elastic wave function, if it belongs to $C^{2}(V)$ and satisfies the time-independent Navier equation (1) at each point of $V$.

\footnotetext{
$\left({ }^{2}\right)$ The symbol " $\leadsto$ " on the top of a vector is used to indicate the unit vector in the same direction.
} 
Definition 2. The exterior elastic wave function $\mathbf{u}: V \rightarrow \mathbf{C}^{3}$ is called an elastic radiation function whenever the longitudinal part $\mathbf{u}^{p}$ and the transverse part $\mathbf{u}^{s}$ of $\mathbf{u}$, as in (3), satisfy the Kupradze radiation conditions (4), (5), uniformly over directions.

In order to facilitate the proof of the main expansion theorem we state and prove the following lemmas.

Lemma 1. The fundamental tensor $\Gamma\left(\mathbf{r}, \mathbf{r}^{\prime}\right)$ can be written in the following form:

$$
\begin{aligned}
\Gamma\left(\mathbf{r}, \mathbf{r}^{\prime}\right)=\frac{f^{p}(R)}{\omega^{2}}\left[k_{p}^{2} \widehat{\mathbf{R}} \otimes\right. & \left.\widehat{\mathbf{R}}-\left(\frac{i k_{p}}{R}-\frac{1}{R^{2}}\right)(\mathbf{I}-3 \widehat{\mathbf{R}} \otimes \widehat{\mathbf{R}})\right] \\
& +\frac{f^{s}(R)}{\omega^{2}}\left[k_{s}^{2}(\mathbf{I}-\widehat{\mathbf{R}} \otimes \widehat{\mathbf{R}})+\left(\frac{i k_{s}}{R}-\frac{1}{R^{2}}\right)(\mathbf{I}-3 \widehat{\mathbf{R}} \otimes \widehat{\mathbf{R}})\right]
\end{aligned}
$$

where

$$
\mathbf{R}=\mathbf{r}-\mathbf{r}^{\prime}=R \hat{\mathbf{R}}, \quad R=\left|\mathbf{r}-\mathbf{r}^{\prime}\right|, \quad \hat{\mathbf{R}}=\frac{\mathbf{r}-\mathbf{r}^{\prime}}{\left|\mathbf{r}-\mathbf{r}^{\prime}\right|}
$$

and

$$
\left.\begin{array}{c}
f^{p}(R)=\frac{e^{i k_{p} R}}{R} \\
f^{s}(R)=\frac{e^{i k_{s} R}}{R}
\end{array}\right\} .
$$

Proof. Set

$$
f(R)=\frac{e^{i k R}}{R}
$$

and use the identity

$$
\nabla_{\mathbf{r}} \otimes \nabla_{\mathbf{r}} f(R)=f^{\prime \prime}(R) \widehat{\mathbf{R}} \otimes \widehat{\mathbf{R}}+\frac{f^{\prime}(R)}{R}(\mathbf{I}-\widehat{\mathbf{R}} \otimes \widehat{\mathbf{R}})
$$

in $(8)$.

Lemma 2. The action of the surface traction operator $T_{\mathbf{r}^{\prime}}$, given by (2), on the fundamental tensor $\Gamma\left(\mathbf{r}, \mathbf{r}^{\prime}\right)$ can be written in the following form:

$$
\begin{aligned}
T_{\mathbf{r}^{\prime}} \boldsymbol{\Gamma}\left(\mathbf{r}, \mathbf{r}^{\prime}\right)=\frac{f^{p}(R)}{\omega^{2}}[ & \left.k_{p}^{2}\left(i k_{p}-\frac{1}{R}\right) \mathbf{A}_{1}+\left(\frac{k_{p}^{2}}{R}+\frac{3 i k_{p}}{R^{2}}-\frac{3}{R^{3}}\right) \mathbf{A}_{3}\right] \\
& -\frac{f^{s}(R)}{\omega^{2}}\left[k_{s}^{2}\left(i k_{s}-\frac{1}{R}\right) \mathbf{A}_{2}+\left(\frac{k_{s}^{2}}{R}+\frac{3 i k_{s}}{R^{2}}-\frac{3}{R^{3}}\right) \mathbf{A}_{3}\right]
\end{aligned}
$$

where

$$
\begin{aligned}
& \mathbf{A}_{1}=-\lambda \hat{\mathbf{r}}^{\prime} \otimes \hat{\mathbf{R}}-2 \mu\left(\hat{\mathbf{r}}^{\prime} \cdot \widehat{\mathbf{R}}\right) \widehat{\mathbf{R}} \otimes \widehat{\mathbf{R}} \\
& \mathbf{A}_{2}=\mu\left[\left(\hat{\mathbf{r}}^{\prime} \cdot \widehat{\mathbf{R}}\right)(\mathbf{I}-2 \widehat{\mathbf{R}} \otimes \widehat{\mathbf{R}})+\widehat{\mathbf{R}} \otimes \hat{\mathbf{r}}^{\prime}\right], \\
& \mathbf{A}_{3}=-2 \mu\left[\left(\hat{\mathbf{r}}^{\prime} \cdot \widehat{\mathbf{R}}\right)(\mathbf{I}-5 \widehat{\mathbf{R}} \otimes \widehat{\mathbf{R}})+\hat{\mathbf{r}}^{\prime} \otimes \widehat{\mathbf{R}}+\widehat{\mathbf{R}} \otimes \hat{\mathbf{r}}^{\prime}\right],
\end{aligned}
$$

and $f^{p}(R), f^{s}(R)$ are given by $(10)$.

Proof. Set

$$
F(R)=f^{s}(R)-f^{p}(R) .
$$


Then the gradient, with respect to $\mathbf{r}^{\prime}$, of the fundamental dyadic $\Gamma\left(\mathbf{r}, \mathbf{r}^{\prime}\right)$ is the following triadic:

$$
\begin{aligned}
\nabla_{\mathbf{r}^{\prime}} \boldsymbol{\Gamma}\left(\mathbf{r}, \mathbf{r}^{\prime}\right)= & -k_{s}^{2} \frac{f^{s^{\prime}}(R)}{\omega^{2}} \widehat{\mathbf{R}} \otimes \mathbf{I} \\
& -\frac{1}{\omega^{2}}\left(\frac{F^{\prime}(R)}{R}\right)^{\prime}\left[\widehat{\mathbf{R}} \otimes \mathbf{I}+\mathbf{I} \otimes \widehat{\mathbf{R}}-\widehat{\mathbf{R}} \otimes \widehat{\mathbf{R}} \otimes \widehat{\mathbf{R}}+\sum_{i=1}^{3} \hat{\mathbf{x}}_{i} \otimes \widehat{\mathbf{R}} \otimes \hat{\mathbf{x}}_{i}\right] \\
& -\frac{R}{\omega^{2}}\left(\frac{F^{\prime}(R)}{R}\right)^{\prime \prime} \widehat{\mathbf{R}} \otimes \widehat{\mathbf{R}} \otimes \widehat{\mathbf{R}},
\end{aligned}
$$

which has the following scalar and vector invariants with respect to the first tensorial product:

$$
\begin{aligned}
& \nabla_{\mathbf{r}^{\prime}} \cdot \boldsymbol{\Gamma}\left(\mathbf{r}, \mathbf{r}^{\prime}\right)=-\frac{k_{s}^{2}}{\omega^{2}} f^{s^{\prime}}(R) \widehat{\mathbf{R}}-\frac{1}{\omega^{2}}\left[4\left(\frac{F^{\prime}(R)}{R}\right)^{\prime}+R\left(\frac{F^{\prime}(R)}{R}\right)^{\prime \prime}\right] \widehat{\mathbf{R}}, \\
& \nabla_{\mathbf{r}^{\prime}} \times \Gamma\left(\mathbf{r}, \mathbf{r}^{\prime}\right)=-\frac{k_{s}^{2}}{\omega^{2}} f^{s^{\prime}}(R) \widehat{\mathbf{R}} \times \mathbf{I} .
\end{aligned}
$$

Moreover, the left contraction of (18) with $\hat{\mathbf{r}}^{\prime}$ provides the identity:

$$
\begin{aligned}
\hat{\mathbf{r}}^{\prime} \cdot \nabla_{\mathbf{r}^{\prime}} \Gamma\left(\mathbf{r}, \mathbf{r}^{\prime}\right)= & -\frac{k_{s}^{2}}{\omega^{2}} f^{s^{\prime}}(R)\left(\hat{\mathbf{r}}^{\prime} \cdot \widehat{\mathbf{R}}\right) \mathbf{I} \\
& -\frac{1}{\omega^{2}}\left(\frac{F^{\prime}(R)}{R}\right)^{\prime}\left[\left(\hat{\mathbf{r}}^{\prime} \cdot \widehat{\mathbf{R}}\right)(\mathbf{I}-\widehat{\mathbf{R}} \otimes \widehat{\mathbf{R}})+\hat{\mathbf{r}}^{\prime} \otimes \widehat{\mathbf{R}}+\widehat{\mathbf{R}} \otimes \hat{\mathbf{r}}^{\prime}\right] \\
& -\frac{R}{\omega^{2}}\left(\frac{F^{\prime}(R)}{R}\right)^{\prime \prime}\left(\hat{\mathbf{r}}^{\prime} \cdot \widehat{\mathbf{R}}\right) \widehat{\mathbf{R}} \otimes \widehat{\mathbf{R}} .
\end{aligned}
$$

Substituting (19), (20), and (21) into the expression for $T_{\mathbf{r}^{\prime}} \boldsymbol{\Gamma}\left(\mathbf{r}, \mathbf{r}^{\prime}\right)$ and using the identity

$$
\hat{\mathbf{r}}^{\prime} \times(\widehat{\mathbf{R}} \times \mathbf{I})=\widehat{\mathbf{R}} \otimes \hat{\mathbf{r}}^{\prime}-\left(\widehat{\mathbf{R}} \cdot \hat{\mathbf{r}}^{\prime}\right) \mathbf{I}
$$

as well as formulae (10) and (17), we arrive, after some long calculations, to the expression (13), with the dyadics $\mathbf{A}_{n}, n=1,2,3$, given by (14)-(16). This proves the lemma.

Let $2 a, a>0$, be the diameter of the scatterer $V^{-}$. We choose the center of the smallest sphere that circumscribes the scatterer as the origin $\mathbf{O}$ and we introduce spherical coordinates with respect to $\mathbf{O}$. Then the following is true.

LEMMA 3. If $\mathbf{u}$ is an elastic radiation function in the domain $V$ then, for every $r>a$,

$$
\mathbf{u}(\mathbf{r})=\frac{a^{2}}{4 \pi \omega^{2}} \sum_{n=1}^{4} \int_{\left|\hat{\mathbf{r}}^{\prime}\right|=1} R^{n-1}\left[f^{p}(R) \mathbf{E}_{n}^{p}\left(\hat{\mathbf{r}}^{\prime}\right)-f^{s}(R) \mathbf{E}_{n}^{s}\left(\hat{\mathbf{r}}^{\prime}\right)\right] d S\left(\hat{\mathbf{r}}^{\prime}\right),
$$

where $\mathbf{E}_{n}^{p}\left(\hat{\mathbf{r}}^{\prime}\right), \mathbf{E}_{n}^{s}\left(\hat{\mathbf{r}}^{\prime}\right)$, for $n=1,2,3,4$, are known functions of the direction of integration $\hat{\mathbf{r}}^{\prime}$, specified by the angular variables $\theta^{\prime}, \varphi^{\prime}$.

Proof. The field $\mathbf{u}$ has the representation (7) and, in view of Betti's third formula [8], the surface $S$ can be deformed to the sphere $r=a$. Then

$$
\mathbf{u}(\mathbf{r})=\frac{a^{2}}{4 \pi} \int_{\left|\mathbf{r}^{\prime}\right|=1}\left[\Psi\left(\mathbf{r}^{\prime}\right) \cdot T_{\mathbf{r}^{\prime}} \boldsymbol{\Gamma}\left(\mathbf{r}, \mathbf{r}^{\prime}\right)-\Gamma\left(\mathbf{r}, \mathbf{r}^{\prime}\right) \cdot T_{\mathbf{r}^{\prime}} \Psi\left(\mathbf{r}^{\prime}\right)\right] d S\left(\hat{\mathbf{r}}^{\prime}\right)
$$


We use Lemma 1 and Lemma 2 to separate the $R$ dependence of $\Gamma\left(\mathbf{r}, \mathbf{r}^{\prime}\right)$ and $T_{\mathbf{r}^{\prime}} \boldsymbol{\Gamma}\left(\mathbf{r}, \mathbf{r}^{\prime}\right)$ from their corresponding angular dependence.

This program leads to the expansion (23), with the direction dependent fields $\mathbf{E}_{n}^{p}$, $\mathbf{E}_{n}^{s}, n=1,2,3,4$, given by

$$
\begin{aligned}
& \mathbf{E}_{1}^{p}\left(\hat{\mathbf{r}}^{\prime}\right)=i k_{p}^{3} \boldsymbol{\Psi} \cdot \mathbf{A}_{1}-k_{p}^{2}(T \boldsymbol{\Psi}) \cdot \widehat{\mathbf{R}} \otimes \widehat{\mathbf{R}}, \\
& \mathbf{E}_{1}^{s}\left(\hat{\mathbf{r}}^{\prime}\right)=i k_{s}^{3} \Psi \cdot \mathbf{A}_{2}+k_{s}^{2}(T \boldsymbol{\Psi}) \cdot(\mathbf{I}-\widehat{\mathbf{R}} \otimes \widehat{\mathbf{R}}), \\
& \mathbf{E}_{2}^{p}\left(\hat{\mathbf{r}}^{\prime}\right)=-k_{p}^{2} \boldsymbol{\Psi} \cdot\left(\mathbf{A}_{1}-\mathbf{A}_{3}\right)+i k_{p}(T \boldsymbol{\Psi}) \cdot(\mathbf{I}-3 \widehat{\mathbf{R}} \otimes \widehat{\mathbf{R}}), \\
& \mathbf{E}_{2}^{s}\left(\hat{\mathbf{r}}^{\prime}\right)=-k_{s}^{2} \boldsymbol{\Psi} \cdot\left(\mathbf{A}_{2}-\mathbf{A}_{3}\right)+i k_{s}(T \boldsymbol{\Psi}) \cdot(\mathbf{I}-3 \widehat{\mathbf{R}} \otimes \widehat{\mathbf{R}}), \\
& \mathbf{E}_{3}^{p}\left(\hat{\mathbf{r}}^{\prime}\right)=3 i k_{p} \Psi \cdot \mathbf{A}_{3}-(T \Psi) \cdot(\mathbf{I}-3 \widehat{\mathbf{R}} \otimes \widehat{\mathbf{R}}), \\
& \mathbf{E}_{3}^{s}\left(\hat{\mathbf{r}}^{\prime}\right)=3 i k_{s} \Psi \cdot \mathbf{A}_{3}-(T \Psi) \cdot(\mathbf{I}-3 \widehat{\mathbf{R}} \otimes \widehat{\mathbf{R}}), \\
& \mathbf{E}_{4}^{p}\left(\hat{\mathbf{r}}^{\prime}\right)=\mathbf{E}_{4}^{s}\left(\hat{\mathbf{r}}^{\prime}\right)=-3 \Psi \cdot \mathbf{A}_{3} .
\end{aligned}
$$

This completes the proof of the lemma.

The following lemma plays a key role in our work and it is a generalization of the corresponding Atkinson's Lemma [1].

LEMMA 4. For every $r \geq r_{0}>a$, the functions $R^{-k} \exp \{i k(R-r)\}, k=1,2,3,4$, where $R=\left|\mathbf{r}-\mathbf{r}^{\prime}\right|$ and $r^{\prime}=a$, are analytic functions in the variable $\rho=a / r$. Their Laurent series expansions converge absolutely and uniformly for $r \geq r_{0}>a$ and all directions $\theta \in[0, \pi], \varphi \in[0,2 \pi)$. Moreover, their expansions can be differentiated term by term with respect to $r, \theta, \varphi$ any number of times and the resulting series are absolutely and uniformly convergent.

Proof. Consider the expression

$$
R=\left|\mathbf{r}-\mathbf{r}^{\prime}\right|=r \sqrt{1-2 \rho \cos \gamma+\rho^{2}}, \quad \rho=\frac{a}{r},
$$

where

$$
\cos \gamma=\hat{\mathbf{r}} \cdot \hat{\mathbf{r}}^{\prime}=\cos \theta \cos \theta^{\prime}+\sin \theta \sin \theta^{\prime} \cos \left(\varphi-\varphi^{\prime}\right) .
$$

Then, for $n=1,2,3,4$,

$$
R^{-n} \exp \{i k(R-r)\}=\left(\frac{\rho}{a}\right)^{n} \frac{\exp \left\{i k a \frac{\sqrt{1-2 \rho \cos \gamma+\rho^{2}}-1}{\rho}\right\}}{\left(1-2 \rho \cos \gamma+\rho^{2}\right)^{n / 2}} .
$$

The function

$$
1-2 \rho \cos \gamma+\rho^{2}=\left(1-\rho e^{i \gamma}\right)\left(1-\rho e^{-i \gamma}\right)
$$

is analytic for $|\rho|<1$ and so is the branch of its square root that has the value +1 at $\rho=0$. Furthermore, the function (35) has no roots inside the unit disc. On the other hand, the function $\rho^{-1}\left[\sqrt{1-2 \rho \cos \gamma+\rho^{2}}-1\right]$ has a removable singularity at $\rho=0$ and hence by redefining its value to be $-\cos \gamma$ at $\rho=0$ it becomes analytic for $|\rho|<1$. Since composition, multiplication, and division preserve analyticity, the four functions given by (34) are all analytic inside the disc $|\rho|<1$. Their expansions in powers of $1 / r$ can be written as

$$
\frac{e^{i k(R-r)}}{R^{n}}=\sum_{m=n}^{+\infty} \frac{a_{n m}\left(\hat{\mathbf{r}}^{\prime}\right)}{r^{m}}, \quad n=1,2,3,4,
$$


for $r \geq r_{0}>a, 0 \leq \theta^{\prime} \leq \pi, 0 \leq \varphi^{\prime}<2 \pi$, where the coefficients $a_{n m}$ depend on the directional variables $\theta^{\prime}$ and $\varphi^{\prime}$. The regularity properties of the series (36) are a consequence of the fact that every power series converges absolutely and uniformly in any compact set which is a subset of its disc of convergence. Moreover, the same is true for the series that results from term by term differentiation. Hence the proof of the lemma.

The expansion theorem can now be stated as follows.

EXPANSION THEOREM. Let $\mathbf{u}(\mathbf{r})$ be an elastic radiation function for the domain $r>a$, where $(r, \theta, \varphi)$ are the spherical coordinates of the observation point $\mathbf{r}$. Then

$$
\mathbf{u}(\mathbf{r})=\frac{e^{i k_{p} r}}{r} \sum_{n=0}^{\infty} \frac{\mathbf{F}_{n}^{p}(\theta, \varphi)}{r^{n}}+\frac{e^{i k_{s} r}}{r} \sum_{n=0}^{\infty} \frac{\mathbf{F}_{n}^{s}(\theta, \varphi)}{r^{n}}
$$

which converges for $r>a$. The series in (37), as well as those obtained by term by term differentiation of any order, converge absolutely and uniformly in the closed domain $r \geq r_{0}>a, \theta \in[0, \pi], \varphi \in[0,2 \pi)$.

Proof. By Lemma 3, the field $\mathbf{u}$ can be written in the form (23), where $f^{p}$ and $f^{s}$ are given by (10). Using Lemma 4, and in particular expansion (36), with the appropriate modifications, in each one of the eight terms in (23) we end up with (37). As far as convergence is concerned, the same line of arguments as in Lemma 4 holds true, and that proves the theorem.

The Expansion Theorem is independent of the boundary conditions on $S$. It rather reflects the radiative character of the field $\mathbf{u}$ outside a sphere that includes the scatterer. In fact, the radiation conditions themselves come out of the first terms of the corresponding series in (37). Therefore, the Expansion Theorem provides the exact form of the radiation function outside the region where the scattering process takes place. The geometry of the scatterer (actual shape of $S$ ), as well as its physical characteristics (boundary conditions) enter the expansion via the orientation dependent coefficients $\mathbf{F}_{n}^{p}$ and $\mathbf{F}_{n}^{s}$.

4. Consequences of the Expansion Theorem. In this section we discuss the consequences of the Expansion Theorem in connection with the behaviour of the angulardependent coefficients in (37). Our goal is to use the fact that $\mathbf{u}$, as it is given by (37), has to satisfy the time-independent equation (1) and find how this is reflected in the coefficients $\mathbf{F}_{n}^{p}$ and $\mathbf{F}_{n}^{s}$.

We write the expansion (37) in the form

$$
\mathbf{u}(\mathbf{r})=\sum_{n=0}^{\infty} f_{n}^{p}(r) \mathbf{F}_{n}^{p}(\theta, \varphi)+\sum_{n=0}^{\infty} f_{n}^{s}(r) \mathbf{F}_{n}^{s}(\theta, \varphi)
$$

where

$$
\left.\begin{array}{rl}
f_{n}^{p}(r) & =\frac{e^{i k_{p} r}}{r^{n+1}} \\
f_{n}^{s}(r) & =\frac{e^{i k_{s} r}}{r^{n+1}}
\end{array}\right\}
$$

$n=0,1,2, \ldots$ 
Lemma 5. If $f_{n}$ is any one of the functions (39) and $\mathbf{F}_{n}$ is a $C^{2}$ vector field defined on the unit sphere of $\mathbf{R}^{3}$ then, for every $n=1,2, \ldots$,

$$
\begin{aligned}
\nabla \otimes \nabla \cdot f_{n}(r) \mathbf{F}_{n}(\theta, \varphi)= & -k^{2} f_{n} \hat{\mathbf{r}} \otimes \hat{\mathbf{r}} \cdot \mathbf{F}_{n} \\
& +\frac{i k}{r} f_{n}[-2(n+1) \hat{\mathbf{r}} \otimes \hat{\mathbf{r}}+\hat{\mathbf{r}} \otimes \mathbf{D}+\mathbf{D} \otimes \hat{\mathbf{r}}] \cdot \mathbf{F}_{n} \\
& +\frac{1}{r^{2}} f_{n}[(n+1)(n+2) \hat{\mathbf{r}} \otimes \hat{\mathbf{r}}-(n+1)(\hat{\mathbf{r}} \otimes \mathbf{D}+\mathbf{D} \otimes \hat{\mathbf{r}}) \\
& +\mathbf{D} \otimes \mathbf{D}-\hat{\mathbf{r}} \otimes \mathbf{D}] \cdot \mathbf{F}_{n}
\end{aligned}
$$

where

$$
\mathbf{D}=\hat{\boldsymbol{\theta}} \frac{\partial}{\partial \theta}+\frac{1}{\sin \theta} \hat{\varphi} \frac{\partial}{\partial \varphi}
$$

and $\hat{\mathbf{r}}, \hat{\boldsymbol{\theta}}, \hat{\varphi}$ are the unit vectors of the spherical coodinate system, and (ii)

$$
\Delta f_{n}(r) \mathbf{F}_{n}(\theta, \varphi)=-k^{2} f_{n} \mathbf{F}_{n}-\frac{2 i k n}{r} f_{n} \mathbf{F}_{n}+\frac{1}{r^{2}}[\mathrm{~B}+n(n+1)] f_{n} \mathbf{F}_{n},
$$

where $\mathbb{B}$ is the Beltrami operator

$$
\mathbb{B}=\mathbf{D} \cdot \mathbf{D}=\frac{1}{\sin \theta} \frac{\partial}{\partial \theta}\left(\sin \theta \frac{\partial}{\partial \theta}\right)+\frac{1}{\sin ^{2} \theta} \frac{\partial^{2}}{\partial \varphi^{2}} .
$$

Proof. By long, but straightforward calculations.

LEMMA 6. Under the hypotheses of Lemma 5

$$
\begin{aligned}
{[\mu \Delta \mathbf{I}} & \left.+(\lambda+\mu) \nabla \otimes \nabla+\omega^{2} \mathbf{I}\right] \cdot f_{n}(r) \mathbf{F}_{n}(\theta, \varphi)=\left[-k^{2} \mathbf{G}_{1}+\omega^{2} \mathbf{I}\right] \cdot f_{n} \mathbf{F}_{n} \\
& +\frac{i k}{r}\left[-2(n+1) \mathbf{G}_{1}+\mathbf{G}_{2}\right] \cdot f_{n} \mathbf{F}_{n}+\frac{1}{r^{2}}\left[(n+1)(n+2) \mathbf{G}_{1}-(n+1) \mathbf{G}_{2}+\mathbf{G}_{3}\right] \cdot f_{n} \mathbf{F}_{n}
\end{aligned}
$$

where

$$
\begin{aligned}
& \mathbf{G}_{1}=\mu \mathbf{I}+(\lambda+\mu) \hat{\mathbf{r}} \otimes \hat{\mathbf{r}}, \\
& \mathbf{G}_{2}=2 \mu \mathbf{I}+(\lambda+\mu)(\mathbf{D} \otimes \hat{\mathbf{r}}+\hat{\mathbf{r}} \otimes \mathbf{D}), \\
& \mathbf{G}_{3}=\mu \mathbf{I} \mathbf{B}+(\lambda+\mu)(\mathbf{D} \otimes \mathbf{D}-\hat{\mathbf{r}} \otimes \mathbf{D}) .
\end{aligned}
$$

Proof. A consequence of Lemma 5.

A key role is played by the following lemma.

LemMA 7. If $\alpha, \beta \in \mathbf{R}$ and $\alpha \neq \beta$ then the countably infinite set

$$
\left\{r^{-(n+1)} e^{i \alpha r}, r^{-(n+1)} e^{i \beta r}\right\}_{n=0}^{\infty}
$$

is linearly independent in the domain $r>a$.

Proof. By definition [5], [11], it is enough to show that every finite subset is linearly independent. A linear combination of a finite subset of our set of functions can be written in the form

$$
e^{i \alpha r} P_{n_{\alpha}}\left(r^{-1}\right)+e^{i \beta r} P_{n_{\beta}}\left(r^{-1}\right)=0
$$

where $P_{n_{\alpha}}$ and $P_{n_{\beta}}$ are polynomials, in the variable $r^{-1}$, of degree $n_{\alpha}$ and $n_{\beta}$ respectively. We assume that (48) is identically zero for $r \in(a,+\infty)$. On the other hand, 
the polynomial $P_{n_{\alpha}}$ has at most $n_{\alpha}$ distinct roots in the complex plane $\mathbf{C}$ and similarly, the number of roots of $P_{n_{\beta}}$ in $\mathbf{C}$ are no more than $n_{\beta}$. Since the functions $e^{i \alpha r}$ and $e^{i \beta r}$ have no roots in $\mathbf{C}$ it follows that all the coefficients of both polynomials are equal to zero. This implies linear independence and proves the lemma.

LemMa 8. (i) The coefficient $\mathbf{F}_{0}^{p}$ in (37) has the radial direction $\hat{\mathbf{r}}$, while the coefficients $\mathbf{F}_{n}^{p}, n=1,2, \ldots$, are given by the recurrence relations

$$
k_{p}^{2}(\lambda+\mu)(\mathbf{I}-\hat{\mathbf{r}} \otimes \hat{\mathbf{r}}) \cdot \mathbf{F}_{1}^{p}=-i k_{p}\left[-2 \mathbf{G}_{1}+\mathbf{G}_{2}\right] \cdot \mathbf{F}_{0}^{p}
$$

and

$$
\begin{aligned}
k_{p}^{2}(\lambda+\mu)(\mathbf{I}-\hat{\mathbf{r}} \otimes \hat{\mathbf{r}}) \cdot \mathbf{F}_{n}^{p}= & -i k_{p}\left[-2 n \mathbf{G}_{1}+\mathbf{G}_{2}\right] \cdot \mathbf{F}_{n-1}^{p} \\
& -\left[(n-1)\left(n \mathbf{G}_{1}-\mathbf{G}_{2}\right)+\mathbf{G}_{3}\right] \cdot \mathbf{F}_{n-2}^{p}
\end{aligned}
$$

for $n=2,3, \ldots$

(ii) The coefficient $\mathbf{F}_{0}^{s}$ is orthogonal to the radial direction $\hat{\mathbf{r}}$, while the coefficients $\mathbf{F}_{n}^{s}, n=1,2, \ldots$, are given by the recurrence relations

$$
k_{s}^{2}(\lambda+\mu) \hat{\mathbf{r}} \otimes \hat{\mathbf{r}} \cdot \mathbf{F}_{1}^{s}=i k_{s}\left[-2 \mathbf{G}_{1}+\mathbf{G}_{2}\right] \cdot \mathbf{F}_{0}^{s}
$$

and

$$
\begin{aligned}
k_{s}^{2}(\lambda+\mu) \hat{\mathbf{r}} \otimes \hat{\mathbf{r}} \cdot \mathbf{F}_{n}^{s}= & i k_{s}\left[-2 n \mathbf{G}_{1}+\mathbf{G}_{2}\right] \cdot \mathbf{F}_{n-1}^{s} \\
& +\left[(n-1)\left(n \mathbf{G}_{1}-\mathbf{G}_{2}\right)+\mathbf{G}_{3}\right] \cdot \mathbf{F}_{n-2}^{s}
\end{aligned}
$$

for $n=2,3, \ldots$

Proof. Substituting the field $\mathbf{u}$, as it is given by (37), into Equation (1) and using Lemmas 6 and 7, we arrive at (49)-(52), as well as the $n=0$ terms of the two series, which give

$$
k_{p}^{2}(\lambda+\mu)(\mathbf{I}-\hat{\mathbf{r}} \otimes \hat{\mathbf{r}}) \cdot \mathbf{F}_{0}^{p}=0
$$

and

$$
k_{s}^{2}(\lambda+\mu) \hat{\mathbf{r}} \otimes \hat{\mathbf{r}} \cdot \mathbf{F}_{0}^{s}=0 .
$$

Relation (53) implies that $\mathbf{F}_{0}^{p}$ is radial, while (54) implies that $\mathbf{F}_{0}^{s}$ is tangential to any sphere with radius greater than $a$. This completes the proof of Lemma 8 .

The leading coefficients $\mathbf{F}_{0}^{p}$ and $\mathbf{F}_{0}^{s}$ are known as scattering amplitudes [4], [22], radiation patterns [14], scattering coefficients [10] and many other names. The fact that $\mathbf{F}_{0}^{p}$ is parallel and that $\mathbf{F}_{0}^{s}$ is perpendicular to the direction of observation $\hat{\mathbf{r}}$ has also been proved by asymptotic methods [2], [4]. Formulae (49), (50) provide the tangential components of the coefficients $\mathbf{F}_{n}^{p}, n=1,2, \ldots$, via the radial radiation pattern $\mathbf{F}_{0}^{p}$. Similarly, formulae (51), (52) provide the radial component of the coefficients $\mathbf{F}_{n}^{s}, n=1,2, \ldots$, via the tangential radiation pattern $\mathbf{F}_{n}^{s}$. In order to be able to express the radial component of $\mathbf{F}_{n}^{p}$, as well as the tangential components of $\mathbf{F}_{n}^{s}$ via the radiation patterns $\mathbf{F}_{0}^{p}$ and $\mathbf{F}_{0}^{s}$, respectively, we assume the following decomposition

$$
\begin{aligned}
& \mathbf{F}_{n}^{p}=\hat{\mathbf{r}} F_{r n}^{p}+\mathbf{F}_{t n}^{p}, \\
& \mathbf{F}_{n}^{s}=\hat{\mathbf{r}} F_{r n}^{s}+\mathbf{F}_{t n}^{s},
\end{aligned}
$$


where $F_{r n}^{p}, F_{r n}^{s}$ are the radial components and $\mathbf{F}_{t n}^{p}, \mathbf{F}_{t n}^{s}$ are the tangential vectors of the corresponding coefficients.

Proposition 1. The radial and the tangential components of the longitudinal coefficients $\mathbf{F}_{n}^{p}, n=1,2, \ldots$, are given in terms of $\mathbf{F}_{0}^{p}=F_{r 0}^{p} \hat{\mathbf{r}}$ by the formulae

$$
\begin{gathered}
2 i k_{p} F_{r 1}^{p}=(\mathbb{B}-2) F_{r 0}^{p}, \\
i k_{p} \mathbf{F}_{t 1}^{p}=\mathbf{D} F_{r 0}^{p}, \\
2 n \frac{\lambda+2 \mu}{\lambda+\mu} k_{p}^{2} F_{r n}^{p}=(\mathbf{D}-2 \hat{\mathbf{r}}) \cdot\left[2 i k_{p}(n-1) \frac{\mu}{\lambda+\mu} \mathbf{F}_{(n-1)}^{p}-\mathbf{L}_{n} \cdot \mathbf{F}_{(n-2)}^{p}\right] \\
-i k_{p}\left[\mathbf{B} F_{r(n-1)}^{p}+\hat{\mathbf{r}} \cdot \mathbf{L}_{n+1} \cdot \mathbf{F}_{(n-1)}^{p}\right], \\
k_{p}^{2} \mathbf{F}_{t n}^{p}=2 i k_{p}(n-1) \frac{\mu}{\lambda+\mu} \mathbf{F}_{t(n-1)}^{p}-(\mathbf{I}-\hat{\mathbf{r}} \otimes \hat{\mathbf{r}}) \cdot \mathbf{L}_{n} \cdot \mathbf{F}_{(n-2)}^{p}-i k_{p} \mathbf{D} F_{r(n-1)}^{p},
\end{gathered}
$$

where

$$
\mathbf{L}_{n}=\frac{\mu}{\lambda+\mu}[(n-1)(n-2)+\mathbb{B}] \mathbf{I}+(\mathbf{D}-n \hat{\mathbf{r}}) \otimes(\mathbf{D}-(n-1) \hat{\mathbf{r}})
$$

for each $n=2,3, \ldots$.

Proof. Decompose the dyadics that appear in the expressions (49) and (50) into their radial and angular parts as follows:

$$
\begin{aligned}
-2 n \mathbf{G}_{1}+\mathbf{G}_{2}= & {[(\lambda+\mu) \hat{\mathbf{r}} \otimes(\mathbf{D}-2 \hat{\mathbf{r}})-2(n-1)(\lambda+2 \mu) \hat{\mathbf{r}} \otimes \hat{\mathbf{r}}] } \\
& +[(\lambda+\mu) \mathbf{D} \otimes \hat{\mathbf{r}}-2(n-1) \mu(\mathbf{I}-\hat{\mathbf{r}} \otimes \hat{\mathbf{r}})]
\end{aligned}
$$

for $n=1,2, \ldots$, and

$$
\begin{aligned}
(n-1) & \left(n \mathbf{G}_{1}-\mathbf{G}_{2}\right)+\mathbf{G}_{3}= \\
& \{\mu \hat{\mathbf{r}} \otimes \hat{\mathbf{r}}[(n-1)(n-2)+\mathbb{B}]-n(\lambda+\mu) \hat{\mathbf{r}} \otimes[\mathbf{D}-(n-1) \hat{\mathbf{r}}]\} \\
& +\{\mu(\mathbf{I}-\hat{\mathbf{r}} \otimes \hat{\mathbf{r}})[(n-1)(n-2)+\mathbb{B}]+(\lambda+\mu) \mathbf{D} \otimes[\mathbf{D}-(n-1) \hat{\mathbf{r}}]\}
\end{aligned}
$$

for $n=2,3, \ldots$ Then introduce the corresponding decomposition (55) and project Eqs. (49), (50) into the direction $\hat{\mathbf{r}}$, to obtain the radial part $F_{r n}^{p}$, and into its orthogonal complement, to obtain the tangential part $\mathbf{F}_{t n}^{p}$. The corresponding projections can be written as

$$
\begin{gathered}
i k_{p} \mathbf{F}_{t 1}^{p}=\mathbf{D} F_{r 0}^{p}, \\
0=-i k_{p}[(\lambda+\mu)(\mathbf{D}-2 \hat{\mathbf{r}})-2(n-1)(\lambda+2 \mu) \hat{\mathbf{r}}] \cdot \mathbf{F}_{(n-1)}^{p} \\
-\{\mu \hat{\mathbf{r}}[(n-1)(n-2)+\mathbf{B}]-n(\lambda+\mu)[\mathbf{D}-(n-1) \hat{\mathbf{r}}]\} \cdot \mathbf{F}_{(n-2)}^{p},
\end{gathered}
$$

and

$$
\begin{aligned}
k_{p}^{2}(\lambda+\mu) & \mathbf{F}_{t n}^{p}= \\
& -i k_{p}[(\lambda+\mu) \mathbf{D} \otimes \hat{\mathbf{r}}-2(n-1) \mu(\mathbf{I}-\hat{\mathbf{r}} \otimes \hat{\mathbf{r}})] \cdot \mathbf{F}_{(n-1)}^{p} \\
& -\{\mu(\mathbf{I}-\hat{\mathbf{r}} \otimes \hat{\mathbf{r}})[(n-1)(n-2)+\mathbf{B}] \\
& +(\lambda+\mu) \mathbf{D} \otimes[\mathbf{D}-(n-1) \hat{\mathbf{r}}]\} \cdot \mathbf{F}_{(n-2)}^{p}
\end{aligned}
$$

for $n=2,3, \ldots$

Equation (66) readily provides the tangential part of $\mathbf{F}_{t n}^{p}$ via $\mathbf{F}_{n-1}^{p}$ and $\mathbf{F}_{n-2}^{p}$. In order to obtain the radial part $F_{r n}^{p}$ also, we use Eq. (65) with $n+1$ instead of $n$, 
substitute $\mathbf{F}_{t n}^{p}$ from (66) and solve with respect to $F_{r n}^{p}$. The same technique is used to obtain (57). The recurrence relations (57)-(61) are compact forms of the final expressions we obtain, after the program is carried out. This concludes the proof of Proposition 1.

Proposition 2. The radial and the tangential components of the transverse coefficients $\mathbf{F}_{n}^{s}, n=1,2, \ldots$, are given in terms of $\mathbf{F}_{0}^{s}=F_{\theta 0}^{s} \hat{\boldsymbol{\theta}}+F_{\varphi 0}^{s} \hat{\varphi}$ by the formulae

$$
\begin{aligned}
i k_{s} F_{r 1}^{s}= & -\mathbf{D} \cdot \mathbf{F}_{0}^{s}, \\
2 i k_{s} \mathbf{F}_{t 1}^{s}= & (\mathbf{I}-\hat{\mathbf{r}} \otimes \hat{\mathbf{r}}) \cdot \mathbf{B} \mathbf{F}_{0}^{s}, \\
k_{s}^{2} F_{r n}^{s}= & i k_{s}\left(\mathbf{K}_{n} \cdot \mathbf{F}_{(n-1)}^{s}\right)+\hat{\mathbf{r}} \cdot \mathbf{L}_{n} \cdot \mathbf{F}_{(n-2)}^{s}, \\
2 n \frac{\mu}{\lambda+\mu} k_{s}^{2} \mathbf{F}_{t n}^{s}= & -i k_{s}(\mathbf{I}-\hat{\mathbf{r}} \otimes \hat{\mathbf{r}}) \cdot \mathbf{L}_{(n+1)} \cdot \mathbf{F}_{(n-1)}^{s} \\
& +\mathbf{D}\left[i k_{s}\left(\mathbf{K}_{n} \cdot \mathbf{F}_{(n-1)}^{s}\right)+\hat{\mathbf{r}} \cdot \mathbf{L}_{n} \cdot \mathbf{F}_{(n-2)}^{s}\right],
\end{aligned}
$$

where

$$
\mathbf{K}_{n}=\mathbf{D}+2 \frac{\mu-n(\lambda+2 \mu)}{\lambda+\mu} \hat{\mathbf{r}}
$$

for $n=2,3, \ldots$ and $\mathbf{L}_{n}$ is given by (61).

Proof. Similar to the proof of Proposition 1.

Propositions 1 and 2 represent an inverse type result. They state that, if the longitudinal radiation pattern $\mathbf{F}_{0}^{p}$ is known, then the whole longitudinal field $\mathbf{u}^{p}$ can be recovered outside the sphere of radius $a$ and, similarly, the transverse field $\mathbf{u}^{s}$, outside the same sphere, can be constructed from the knowledge of the transverse radiation pattern $\mathbf{F}_{0}^{s}$. We summarize this property in the following theorem.

Correspondence Theorem. There exists a one-to-one correspondence between the longitudinal radiation fields and the longitudinal radiation patterns on the one hand, and between the transverse radiation fields and the transverse radiation patterns on the other.

Proof. Given any longitudinal radiation function $\mathbf{u}^{p}$, construct the first series in the right-hand side of (37) and pick up the unique longitudinal radiation pattern $\mathbf{F}_{0}^{p}$ as the coefficient of $r^{-1} \exp \left\{i k_{p} r\right\}$ in the first term of the series. Conversely, given any longitudinal radiation pattern $\mathbf{F}_{0}^{p}$, use formulae (57)-(60) to evaluate all the longitudinal coefficients $\mathbf{F}_{n}^{p}, n=1,2, \ldots$. The unique field $\mathbf{u}^{p}$ that corresponds to $\mathbf{F}_{0}^{p}$ is the first series in the right-hand side of (37). In a similar way, we can show the corresponding result for the transverse field, and this completes the proof.

Using asymptotic methods, it is proved in [4] that the leading term approximation of the scattered field in the radiation zone has the form

$$
\mathbf{u}(\mathbf{r})=g_{r}(\hat{\mathbf{r}}, \hat{\mathbf{k}}) \hat{\mathbf{r}} \frac{e^{i k_{p} r}}{i k_{p} r}+g_{\theta}(\hat{\mathbf{r}}, \hat{\mathbf{k}}) \hat{\boldsymbol{\theta}} \frac{e^{i k_{s} r}}{i k_{s} r}+g_{\varphi}(\hat{\mathbf{r}}, \hat{\mathbf{k}}) \hat{\varphi} \frac{e^{i k_{s} r}}{i k_{s} r}+O\left(\frac{1}{r^{2}}\right), \quad r \rightarrow \infty,
$$

where the normalized spherical scattering amplitudes $g_{r}, g_{\theta}, g_{\varphi}$ are given in terms of surface integrals over the surface of the scatterer (formulae (46)-(53) in [4]). 
Therefore, the radiation patterns

$$
\begin{aligned}
& \mathbf{F}_{0}^{p}=\frac{g_{r}(\hat{\mathbf{r}}, \hat{\mathbf{k}})}{i k_{p}} \hat{\mathbf{r}}, \\
& \mathbf{F}_{0}^{s}=\frac{g_{\theta}(\hat{\mathbf{r}}, \hat{\mathbf{k}})}{i k_{s}} \hat{\boldsymbol{\theta}}+\frac{g_{\varphi}(\hat{\mathbf{r}}, \hat{\mathbf{k}})}{i k_{s}} \hat{\boldsymbol{\varphi}}
\end{aligned}
$$

are given in terms of surface integrals that incorporate both the geometry of the scatterer, via the surface of integration, as well as the physical characteristics of the scattering region, via the values of the displacement and the traction fields on the boundary of the scatterer. Using (57), (58) the $\mathbf{F}_{1}^{p}$ coefficient is given by

$$
\begin{aligned}
2 k_{p}^{2} F_{r 1}^{p} & =-(\mathbb{B}-2) g_{r}(\hat{\mathbf{r}}, \hat{\mathbf{k}}), \\
k_{p}^{2} \mathbf{F}_{t 1}^{p} & =-\mathbf{D} g_{r}(\hat{\mathbf{r}}, \hat{\mathbf{k}}),
\end{aligned}
$$

and (59), (60) can give any coefficient $\mathbf{F}_{n}^{p}$ in terms of the radial scattering amplitude $g_{r}(\hat{\mathbf{r}}, \hat{\mathbf{k}})$. Similarly, (67), (68) imply

$$
\begin{aligned}
k_{s}^{2} F_{r 1}^{s} & =\mathbf{D} \cdot\left[g_{\theta}(\hat{\mathbf{r}}, \hat{\mathbf{k}}) \hat{\boldsymbol{\theta}}+g_{\varphi}(\hat{\mathbf{r}}, \hat{\mathbf{k}}) \hat{\varphi}\right], \\
2 k_{s}^{2} \mathbf{F}_{t 1}^{s} & =-(\mathbf{I}-\hat{\mathbf{r}} \otimes \hat{\mathbf{r}}) \cdot \mathbb{B}\left[g_{\theta}(\hat{\mathbf{r}}, \hat{\mathbf{k}}) \hat{\boldsymbol{\theta}}+g_{\varphi}(\hat{\mathbf{r}}, \hat{\mathbf{k}}) \hat{\varphi}\right],
\end{aligned}
$$

while (69), (70) give any other coefficient $\mathbf{F}_{n}^{p}$ in terms of $g_{\theta}(\hat{\mathbf{r}}, \hat{\mathbf{k}})$ and $g_{\varphi}(\hat{\mathbf{r}}, \hat{\mathbf{k}})$.

5. Acoustic and electromagnetic waves. The case of acoustic waves can be recovered from the elastic problem if we set $\lambda=-\mu$, in order to reduce the Navier equation to the classical wave equation, and restrict attention to wave functions of the form $\mathbf{u}=u \mathbf{k}$, where $\mathbf{k}$ is a constant propagation vector. More precisely, setting $\lambda=-\mu$ into the radial recurrence formula (65) we obtain

$$
2 i k n \hat{\mathbf{r}} \cdot \mathbf{F}_{n}=\hat{\mathbf{r}} \cdot[n(n-1)+\mathbb{B}] \mathbf{F}_{(n-1)},
$$

which in view of the scalar restriction $\mathbf{F}_{n}=f_{n} \mathbf{k}$ takes the form

$$
2 i k n f_{n}=[n(n-1)+\mathbb{B}] f_{(n-1)}, \quad n=1,2, \ldots,
$$

which is exactly Eq. (5) in Wilcox's paper for acoustic waves [24].

The electromagnetic case comes out of the transverse radiation field $\mathbf{u}^{s}$, for which $\hat{\mathbf{r}} \cdot \mathbf{F}_{0}=0$, which reflects the solenoidal character of the electric and the magnetic field in free space. Specifically, setting $\lambda=-\mu$ into (67), (69) and the equation for the transverse field corresponding to (66), we obtain

$$
\begin{aligned}
i k F_{r 1} & =-\mathbf{D} \cdot \mathbf{F}_{0}, \\
2 i k n F_{r n} & =\hat{\mathbf{r}} \cdot[n(n-1)+\mathbb{B}] \mathbf{F}_{(n-1)}, \\
2 i k n(\mathbf{I}-\hat{\mathbf{r}} \otimes \hat{\mathbf{r}}) \cdot \mathbf{F}_{n} & =(\mathbf{I}-\hat{\mathbf{r}} \otimes \hat{\mathbf{r}}) \cdot[n(n-1)+\mathbb{B}] \mathbf{F}_{(n-1)} .
\end{aligned}
$$

The condition $\Delta \cdot \mathbf{u}=0$ implies

$$
i k F_{r n}=n F_{r(n-1)}-\mathbf{D} \cdot \mathbf{F}_{(n-1)} .
$$

Also, the spherical decomposition of $\mathbf{B F}$ yields

$$
\mathbf{B F}=\left[\mathrm{B} F_{r}+2 F_{r}-2 \mathbf{D} \cdot \mathbf{F}\right] \hat{\mathbf{r}}+\left[\mathrm{B} F_{\theta}+D_{\theta} \mathbf{F}\right] \hat{\boldsymbol{\theta}}+\left[\mathbb{B} F_{\varphi}+D_{\varphi} \mathbf{F}\right] \hat{\varphi}
$$


where the differential operators $D_{\theta}, D_{\varphi}$, used by Wilcox [25], are given by

$$
\begin{aligned}
& D_{\theta} \mathbf{F}=2 \frac{\partial F_{r}}{\partial \theta}-\frac{1}{\sin ^{2} \theta} F_{\theta}-2 \frac{\cos \theta}{\sin ^{2} \theta} \frac{\partial F_{\varphi}}{\partial \varphi}, \\
& D_{\varphi} \mathbf{F}=\frac{2}{\sin \theta} \frac{\partial F_{r}}{\partial \varphi}+2 \frac{\cos \theta}{\sin ^{2} \theta} \frac{\partial F_{\theta}}{\partial \varphi}-\frac{1}{\sin ^{2} \theta} F_{\varphi} .
\end{aligned}
$$

In view of (85), Eqs. (82) and (84) imply

$$
2 i k n F_{r(n+1)}=[n(n-1)+\mathbb{B}] F_{r n}, \quad n=1,2, \ldots
$$

while the $\hat{\theta}$ and $\hat{\varphi}$ projections of (83) yield

$$
\begin{aligned}
& 2 i k n F_{\theta n}=[n(n-1)+\mathbb{B}] F_{\theta(n-1)}+D_{\theta} \mathbf{F}_{(n-1)}, \\
& 2 i k n F_{\varphi n}=[n(n-1)+\mathbb{B}] F_{\varphi(n-1)}+D_{\varphi} \mathbf{F}_{(n-1)},
\end{aligned}
$$

for $n=1,2, \ldots$ Equations (81), (88), (89), and (90) are exactly the equations for the corresponding electromagnetic problem given by Wilcox, in page 124 of [24].

Both for the acoustic and the electromagnetic case, the recurrence relation that determines the coefficients in the corresponding expansions, in terms of the radiation patterns, are of the second order. On the other hand, the coefficients of the expansion of an elastic radiation function are determined via recurrence formulae of the third order. This is a consequence of the higher complexity of the Navier equation as it compares to the classical wave equation.

\section{REFERENCES}

[1] F. V. Atkinson, On Sommerfeld's Radiation Condition, Phil. Mag. Series 7, 40, 645-651 (1949)

[2] P. J. Barratt, W. D. Collins, The Scattering Cross-Section of an Obstacle in an Elastic Solid for Plane Harmonic Waves, Proc. Camb. Philos. Soc. 61, 969-981, (1965)

[3] D. Colton, R. Kress, Integral Equation Methods in Scattering Theory, John Wiley, New York, 1983

[4] G. Dassios, K. Kiriaki, The Low-Frequency Theory of Elastic Wave Scattering, Quart. Appl. Math. 42, 225-248 (1984)

[5] N. Dunford, J. T. Schwartz, Linear Operators I, Interscience, New York, 1958

[6] F. John, Partial Differential Equations, fourth edition, Springer-Verlag, New York, 1983

[7] S. N. Karp, A Convergent Farfield Expansion for Two-Dimensional Radiation Functions, C. P. A. M. 14, 427-434 (1961)

[8] V. D. Kupradze, Dynamical Problems in Elasticity, in Progress in Solid Mechanics, North-Holland, Amsterdam, 1963

[9] V. D. Kupradze, Three-dimensional Problems of the Mathematical Theory of Elasticity and Thermoelasticity, North-Holland, Amsterdam, 1979

[10] P. D. Lax, R. S. Phillips, Scattering Theory, Academic Press, New York, 1967

[11] L. A. Liusternik, V. J. Sobolev, Elements of Functional Analysis, Ungar, New York, 1961

[12] J. C. Maxwell, Treatise on Electricity and Magnetism, 2 vols., third edition, Dover, New York, 1954

[13] C. Müller, Die Grundzüge einer Mathematischen Theorie Elektromagnetischer Schwingungen, Archiv. der Math. 1, 296-302 (1948-49)

[14] C. Müller, Radiation Patterns and Radiation Fields, J. Rat. Mech. Anal. 4, 235-246 (1955)

[15] J. W. S. Rayleigh, On the incidence of aerial and electrical waves upon small obstacles in the form of ellipsoids, of elliptic cylinders, and on the passage of electric waves through a circular aperture in a conducting screen, Phil. Mag. 44, 28-52 (1897)

[16] S. Silver, Microwave Antenna Theory and Design, M. I. T. Rad. Lab., McGraw-Hill, New York, 1949

[17] A. Sommerfeld, Die Greensche Funktion der Schwingungsgleichung, Jahr. Der Deut. Math. Ver. 21, 309-353 (1912)

[18] J. J. Stoker, Some Remarks on Radiation Conditions, Proc. Symp. Appl. Math. A. M. S. 5, 97-102 (1954) 
[19] J. J. Stoker, On Radiation Conditions, C. P. A. M. 9, 577-595 (1956)

[20] J. A. Stratton, L. J. Chu, Diffraction Theory of Electromagnetic Waves, Phys. Rev. 56, 99-107 (1939)

[21] C. Truesdell, Mechanics of Solids II, Encyclopedia of Physics, Springer-Verlag, Berlin, 1972

[22] V. Twersky, Multiple scattering by arbitrary configurations in three dimensions, J. M. P. 3, 83-91 (1962)

[23] V. Twersky, Rayleigh Scattering, Appl. Opt. 3, 1150-1162 (1964)

[24] C. H. Wilcox, A Generalization of Theorems of Rellich and Atkinson, Proc. Amer. Math. Soc. 7, 271-276 (1956)

[25] C. H. Wilcox, An Expansion Theorem for Electromagnetic Fields, C. P. A. M. 9, 115-134 (1956)

[26] C. H. Wilcox, Spherical Means and Radiation Conditions, A. R. M. A. 3, 133-148 (1959) 